\title{
A Min-Plus Derivation of the Fundamental Car-Traffic Law
}

\author{
Pablo A. Lotito ${ }^{\dagger *}$, Elina M. Mancinelli ${ }^{\ddagger}{ }^{\dagger}$ and Jean-Pierre Quadrat ${ }^{\ddagger}$ \\ pablo.lotito, elina.mancinelli, jean-pierre.quadrat@inria.fr \\ † GRETIA-INRETS, 2 Av. G. Malleret-Joinville - 94114 Arccueil France \\ †INRIA, Domaine de Voluceau - Rocquencourt - B.P. 10578153 Le Chesnay Cedex France \\ $\natural$ CONICET, Argentina
}

\begin{abstract}
We give deterministic and stochastic models of the traffic on a circular road without overtaking. From this model the mean speed is derived as an eigenvalue of the min-plus matrix describing the dynamics of the system in the deterministic case and as the Lyapunov exponent of a min-plus stochastic matrix in the stochastic case. The eigenvalue and the Lyapunov exponent are computed explicitly. From these formulae we derive the fundamental law that links the flow to the density of vehicles on the road. Numerical experiments using the MAXPLUS toolbox of SCILAB confirm the theoretical results obtained.
\end{abstract}

\section{INTRODUCTION}

For simple traffic models a well known relation exists between the flow and the density of vehicles called Fundamental Traffic Law. This law has been studied empirically and theoretically using exclusion processes (see for example [5], [6], [7], [3], [12], [8]) and cellular automata (see [1]).

In this work we analyze the simplest deterministic and stochastic traffic models using the so called min-plus algebra. Within this algebra the equations of the dynamics become linear and the eigenvalue or the Lyapunov exponent of the corresponding min-plus matrix gives the mean speed from which we easily derive the density-flow relation.

The traffic model consists of $N$ cars on a circular road of unitary length. In the deterministic case all cars want to move at a common desired given velocity $\nu$, and must respect a safety distance of $\sigma$ with respect to the car ahead. In the stochastic model the cars choose their velocities randomly and independently between two possible values $\eta$ and $\nu$ (with $\eta<$ $\nu)$, respectively with probabilities $(\mu, \lambda)$. We consider here only the case where the cars are not allowed to overtake other cars.

First, in the deterministic case, the fundamental law is derived from the explicit computation of the min-plus eigenvalue of the matrix describing the dynamics of the system.

Next we study the stochastic model showing that the average speed is the Lyapunov exponent of a stochastic min-plus matrix. In general, it is very difficult to compute a Lyapunov exponent. In our case, it is possible to characterize completely the stationary regime and from this characterization to obtain the Lyapunov exponent. The fundamental traffic law is then easily derived from this result.

The analysis of the deterministic model in terms of eigenvalues of a maxplus matrix is new, but the model and the

* Work done during the sojourn of the first author at Inria-Rocquencourt results are very close ${ }^{1}$ to [3]. In Nagel-Hermann [11] more realistic deterministic models are studied. The state is defined by the vehicle position and its speed instead of only the position. Nevertheless we obtain also a typical hat-shaped fundamental traffic law. This fact suggests that the acceleration is not fundamental in first order approximations. In fact, in Nagel-Hermann we see that in the stationary regime, for the parallel updating rule (the one used here), the system reaches the maximal allowed speed.

The stochastic model proposed is new. Its interest is mainly theoretical since the traffic law obtained is a smoothed version of the hat shape obtained in the deterministic case. The complete analysis can be done only in the simple case when the speed, which is random, can take only two values and when the size of the vehicles is zero. But numerical experiments show that improving the model of speeds and giving a nonzero size to the cars has a negligible influence. Moreover, the analysis in the oversimplified but feasible case used here is qualitatively very informative. The more realistic stochastic model of Nagel-Schreckenberg [12] gives the same kind of traffic law, but the analysis can be done only by numerical experiments. Derrida in [5], [6], [7] gives a complete theoretical analysis of an exclusion stochastic process modelling of traffic which is different from the one proposed here (a vehicle is characterized only by its position and can jump ahead with a given probability if the position ahead is free). In the Derrida model, the process is ergodic and the invariant probabilistic measure can be computed explicitly. Here the process is not ergodic. But we can characterize the stationary regimes and determine completely the invariant measures.

\section{Deterministic Modelling}

We consider $N$ cars moving on a one-way circular road of length 1 . Each car, indexed by $n=1, \cdots, N$, has a desired speed $\nu$, a size 0 , and must respect a security distance $\sigma$ with the car ahead $(N \sigma \leq 1)$. A discrete time dynamic model is used where, at each unitary time step $t$, the driver tries to cover the distance $\nu$ taking into account that it cannot overtake the car ahead. The total distance covered at time $t$ by car $n$ is denoted $x_{n}^{t}$. In order to determine the dynamics of the system, we have to know at what precise instant the safety distances have to be verified. We consider two cases :

\footnotetext{
${ }^{1}$ In our model there are no cells, but the Blank's cell model is also maxplus linear and can be analyzed by the same method and gives the same traffic law.
} 
i) The move of the driver ahead is anticipated (at time $t$ the driver $n$ knows the position that will have the car ahead at time $t+1$ ). Having in mind that the road is circular and that its length is one ${ }^{2}$, the covered distances are given by :

$$
x_{n}^{t+1}= \begin{cases}\min \left(x_{n}^{t}+\nu, x_{n+1}^{t+1}-\sigma\right), & \text { if } n<N, \\ \min \left(x_{N}^{t}+\nu, x_{1}^{t+1}+1-\sigma\right), & \text { if } n=N .\end{cases}
$$

ii) The move of the driver ahead is not anticipated. The distances covered by the cars are :

$$
x_{n}^{t+1}= \begin{cases}\min \left(x_{n}^{t}+\nu, x_{n+1}^{t}-\sigma\right), & \text { if } n<N, \\ \min \left(x_{N}^{t}+\nu, x_{1}^{t}+1-\sigma\right), & \text { if } n=N .\end{cases}
$$

For these two models we will derive a relation between the car density and the average car flow, that will correspond to the fundamental traffic law in traffic theory.

\section{Min-Plus Algebra}

To derive the fundamental traffic law we need to compute the eigenvalue of a min-plus matrix describing the dynamics of the traffic system. In this section we present the principal definitions and properties of the min-plus algebra. The reader is referred to [2] for an in-depth treatment of the subject. A min-plus algebra is defined by the set $\mathbb{R} \cup\{+\infty\}$ together with the operations min (denoted by $\oplus$ ) and + (denoted by $\otimes)$. The element $\varepsilon=+\infty$ satisfies $\varepsilon \oplus x=x$ and $\varepsilon \otimes x=\varepsilon(\varepsilon$ acts as zero). The element $e=0$ satisfies $e \otimes x=x$ ( $e$ is the identity). The main difference with respect to the conventional algebra is that $x \oplus x=x$ (idempotency). We denote $\mathbb{R}_{\text {min }}=$ $(\mathbb{R} \cup\{+\infty\}, \oplus, \otimes)$ this structure. $\mathbb{R}_{\min }$ is a special instance of dioid (semiring with idempotent addition).

This min-plus structure on scalars induces a dioid structure on square matrices with matrix product $A \otimes B$, for two compatible matrices with entries in $\mathbb{R}_{\min }$, defined by $(A \otimes X)_{i k}=\min _{j}\left(A_{i j}+B_{j k}\right)$, where the unit matrix is denoted $\mathrm{E}$. We associate to a square matrix $A$ a precedence graph $\mathcal{G}(A)$ where the nodes correspond to the columns (or the rows) of the matrix $A$ and the arcs to the nonzero entries (the weight of the arc $(i, j)$ being the non zero entry $\left.A_{j i}\right)$. We define $|p|_{w}$ the weight of a path $p$ in $\mathcal{G}(A)$ as the sum of the weights of the arcs composing the path. The arc number of the path $p$ is denoted $|p|_{l}$. We will use the three fundamental results resumed in the following Proposition (see [2]):

Proposition 1: Let $A$ be a $(N \times N)-\mathbb{R}_{\min }$-matrix, and $\mathcal{C}$ the set of circuits of $\mathcal{G}(A)$. We have :

i) If the weights of all the circuits are positive, the equation $x=A \otimes x \oplus b$ admits a unique solution $x=A^{*} \otimes b$ where $A^{*}=E \oplus A \oplus \cdots \oplus A^{N-1} \oplus \cdots=E \oplus A \oplus \cdots \oplus A^{N-1}$.

ii) If $\mathcal{G}(A)$ is strongly connected, the matrix $A$ admits a unique eigenvalue $\lambda \in \mathbb{R}_{\min }$ :

$$
\exists x \in \mathbb{R}_{\min }^{N}: A \otimes x=\lambda \otimes x \text { with } \lambda=\min _{c \in \mathcal{C}} \frac{|c|_{w}}{|c|_{l}} ;
$$

and the min-plus linear dynamic system $X^{t+1}=A \otimes X^{t}$ is asymptotically periodic:

$$
\exists T, K: \forall k \geq K: A^{k+T}=\lambda^{T} \otimes A^{k} .
$$

\section{The Fundamental Traffic Law in the Deterministic Non ANTicipative CASE}

Using the min-plus notation, the dynamics of the traffic in the non anticipative case given by equation (2) may be written in scalar form as follows:

$$
x_{n}^{t+1}= \begin{cases}\nu \otimes x_{n}^{t} \oplus(-\sigma) \otimes x_{n+1}^{t}, & \text { if } n<N, \\ \nu \otimes x_{N}^{t} \oplus(1-\sigma) \otimes x_{1}^{t}, & \text { if } n=N .\end{cases}
$$

In vectorial form, defining $X^{t}=\left[x_{1}^{t}, \ldots, x_{N}^{t}\right]^{\prime}$, we have

$$
X^{t+1}=A \otimes X^{t},
$$

with

$$
A=\left[\begin{array}{cccc}
\nu & -\sigma & & \\
& \ddots & \ddots & \\
& & \ddots & -\sigma \\
1-\sigma & & & \nu
\end{array}\right]
$$

where the missing entries are $\varepsilon$. The precedence graph associated with $A$ is given in Figure 1.

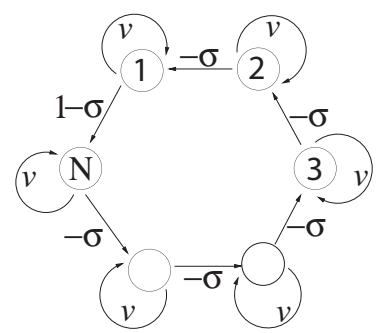

Fig. 1. Precedence Graph of $A$.

In order to use the results given in the Proposition 1 we have to compute the circuits of the graph. The elementary circuits are the loops, of weight $\nu$, and the complete circuit weighting $1-N \sigma$. Using the eigenvalue formula (3), the eigenvalue of $A$ is:

$$
\lambda=\min \left(\nu, \frac{1-N \sigma}{N}\right) .
$$

Considering that the minimal space needed by a car on the road is $\sigma$, the car density $d$ is $N \sigma$ divided by the length of the road, taken equal to 1 , therefore $d=N \sigma$. The average flow is equal to the car density times the average speed, that is $f=\lambda N \sigma$. Then, replacing in (6) we obtain the fundamental traffic law :

$$
f=\min \{\nu d, \sigma(1-d)\} .
$$

Therefore, using this min-plus model, we find again the results presented in [3].

${ }^{2}$ This explains the " $+1 "$ in (1) and (2). 


\section{The Fundamental Traffic LaW in the Deterministic ANTICIPATIVE CASE}

Using min-plus notation, the dynamics of the traffic in the anticipative case may be written

$$
X^{t+1}=A \otimes X^{t+1} \oplus B \otimes X^{t},
$$

where

$$
A=\left[\begin{array}{cccc}
\varepsilon & -\sigma & & \\
& \varepsilon & \ddots & \\
& & \ddots & -\sigma \\
1-\sigma & & & \varepsilon
\end{array}\right], B=\left[\begin{array}{cccc}
\nu & & & \\
& \ddots & & \\
& & \ddots & \\
& & & \nu
\end{array}\right] .
$$

This is an implicit system, to obtain an explicit system we have to compute $A^{*}$ (see Proposition 1-i or [2]). The existence of $A^{*}$ is verified if and only if there is no circuit with negative weight in $\mathcal{G}(A)$, that is, if $1-N \sigma \geq 0$, which is true by assumption. This condition means that there is enough place on the road for the $N$ cars. The explicit form of the equation is :

$$
X^{t+1}=A^{*} \otimes B \otimes X^{t} .
$$

The mean speed of the cars is the $\mathbb{R}_{\min }$ eigenvalue of $A^{*} \otimes B$ which can be easily verified to be equal to $\nu$, therefore in this case, the fundamental traffic law is given by $f=\nu d$. This is an involved application of Theorem 3.28 in [2], nevertheless the result can be guessed without any computation. Indeed, in this deterministic anticipative case, all the cars can move with speed $\nu$, (at the initial time the cars respect the security distance and they can move all together at speed $\nu$ respecting the safety distance).

\section{Stochastic Modelling}

Now we suppose that at each unitary time step $t$, each driver $n$ chooses his desired speed $v_{n}^{t}$ independently and randomly between $\{\eta, \nu\}$ with probabilities $\{\mu, \lambda\}, \eta \leq \nu$. That is, the random variables $\left\{v_{n}^{t}\right\}$, with $n=1, \cdots, N$ and $t \in \mathbb{N}$, are i.i.d. Bernoulli random variables. We suppose that ${ }^{3}$ : (a) $\eta=0$, (b) that the safety distance is 0 (this means that two cars may be in the same position), (c) the drivers may anticipate the move of the car ahead. Then, the dynamics of the system is given by :

$$
x_{n}^{t+1}= \begin{cases}\min \left(v_{n}^{t}+x_{n}^{t}, x_{n+1}^{t+1}\right), & \text { if } n<N, \\ \min \left(v_{N}^{t}+x_{N}^{t}, 1+x_{1}^{t+1}\right), & \text { if } n=N .\end{cases}
$$

This system is still linear in the min-plus algebra but now it is stochastic. Within this algebra the formula (9) becomes

$$
x_{n}^{t+1}= \begin{cases}v_{n}^{t} \otimes x_{n}^{t} \oplus x_{n+1}^{t+1}, & \text { if } n<N, \\ v_{N}^{t} \otimes x_{N}^{t} \oplus 1 \otimes x_{1}^{t+1}, & \text { if } n=N .\end{cases}
$$

\footnotetext{
${ }^{3}$ The assumption (a) is justified by the standard change of variables $x=x^{\prime}+\eta t^{\prime}, t^{\prime}=t$. The assumption (b) allows us to obtain interesting mathematical results . The more general case $(\sigma \neq 0)$ can be analogously modelled (see section $\mathrm{X}$ ) and numerical experiments have shown that the qualitative results are similar. The assumption (c) is more realistic and can be analyzed mathematically but the non anticipative case is easier to analyze and gives the same kind of fundamental car-traffic laws.
}

Defining: $X^{t}=\left[x_{1}^{t}, \ldots, x_{N}^{t}\right]^{\prime}$,

$A=\left[\begin{array}{cccc}\varepsilon & e & & \\ & \ddots & \ddots & \\ & & \ddots & e \\ 1 & & & \varepsilon\end{array}\right], B^{t}=B\left(v^{t}\right)=\left[\begin{array}{cccc}v_{1}^{t} & & & \\ & v_{2}^{t} & & \\ & & \ddots & \\ & & & v_{N}^{t}\end{array}\right]$,

where the missing entries are $\varepsilon$, we can rewrite the equations more compactly as

$$
X^{t+1}=A \otimes X^{t+1} \oplus B^{t} \otimes X^{t} .
$$

In our case $A^{*}$ is easy to compute

$$
A^{*}=\left[\begin{array}{cccc}
e & e & \cdots & e \\
1 & \ddots & & \vdots \\
\vdots & \ddots & \ddots & e \\
1 & \cdots & 1 & e
\end{array}\right]
$$

Then,

$$
X^{t+1}=C^{t} \otimes X^{t},
$$

with $C^{t}=A^{*} \otimes B^{t}$.

Using the fact that the matrices $C^{t}$ are all irreducible (because there are no zero entries in $C^{t}$ ) we know by Corollary 7.31 of [2] that :

$$
\lim _{t} x_{n}^{t} / t=\bar{v}, \quad \forall n
$$

where $\bar{v}$ is called the Lyapunov exponent of the stochastic minplus matrix $C$ (with $\left(C^{t}\right)_{t \in \mathbb{N}}$, independent samples of $C$ ).

In general, there is not a known method to compute explicitly the Lyapunov exponent. Explicit formulæ involving computation of expectations are given in [10], but there is no way to compute explicitly these expectations. Nevertheless, here we are able to characterize the stationary regime of $X^{t}$. This allows us to compute explicitly the expectation appearing in $\bar{v}$.

\section{JAM REGIME}

In order to represent graphically the system state we use the diagrams shown on Figures 2 and 3 where :

i) each segment outside the outer ring has a length proportional to the number of cars in that position;

ii) the black (blue) [resp. grey (green)] length of segments between the two rings are proportional to the number of cars with desired speed 0 [resp. $\nu$ ];

In Figure 2 we show the evolution of the system for 100 cars with speeds 0 and $\nu=1 / 3$.

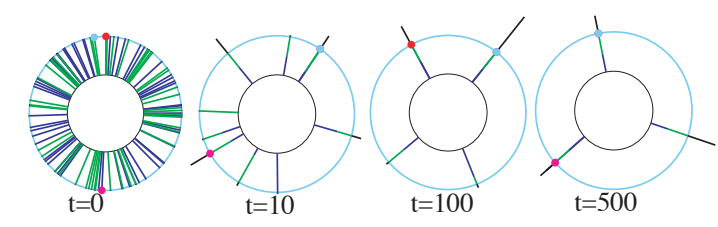

Fig. 2. Evolution of the system with 100 cars and $\nu=1 / 3$.

In Figure 3 we show the evolution of the system for 50 cars with speeds 0 and $\nu=0.3$. 


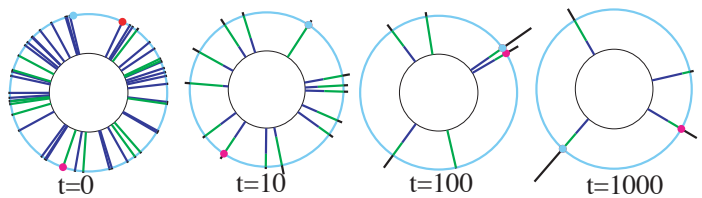

Fig. 3. Evolution of the system with 50 cars and $\nu=0.3$.

Definition 1: We call state the set of positions of the cars on the circle. We call jam state, a state where the cars are concentrated in $k$ clusters, possibly empty, where $k=\left\lceil\frac{1}{\nu}\right\rceil$ is the upper round of $1 / \nu$. The positions of the clusters are given by $\left\{\pi_{1}, \cdots \pi_{k}\right\}$ with $\pi_{i+1}-\pi_{i}=\nu$ for $i=1, \ldots, k-1$. In such a jam state the distance between two clusters is $\nu$ except for at most one pair where the distance is $\gamma=1-(k-1) \nu$. When $1 / \nu \in \mathbb{N}$ we say that the jam state is regular, and the distance between all the clusters is $\nu$.

Definition 2: When for all $t \geq T$ the system stays in a jam state we say that after $T$ the system is in a jam regime.

Proposition 2: A jam state is characterized by $\delta(x)=0$, where

$$
\begin{gathered}
\delta(x)=\min _{h=1, \cdots, N}\left(\sum_{\substack{j=1, \cdots, N \\
j \neq h}}\left\{x_{j+1}-x_{j}\right\}\right) \\
\{x\}=x-\nu\left\lfloor\frac{x}{\nu}\right\rfloor .
\end{gathered}
$$

For non jam states we have $\delta(x)>0$. Moreover

$$
\delta\left(X^{T}\right)=0 \Rightarrow \delta\left(X^{t}\right)=0, \forall t \geq T,
$$

that is, after reaching a jam state the system remains in a jam regime.

Proof: It is easy to see that $\delta(x)=0$ for a jam state $x$. The question is then to show the converse. Let us suppose that $\delta(x)=0$ by definition of $\delta$ there is an $h^{*}$ such that

$$
\sum_{j \neq h^{*}}\left\{x_{j+1}-x_{j}\right\}=0
$$

we can suppose without loss of generality that $h^{*}=N$, then for every $j \neq N, x_{j+1}-x_{j}$ is a multiple of $\nu$. Defining $k=\left\lceil\frac{1}{\nu}\right\rceil$, and considering that

$$
1 \geq x_{N}-x_{1}=\sum_{j=1}^{N-1} x_{j+1}-x_{j},
$$

we have that there are at most $k-1$ non zero terms $j_{1}, \ldots j_{k-1}$ which define the $k-1$ cluster in position $\pi_{1}=x_{j_{1}}, \ldots, \pi_{k-1}=$ $x_{j_{k-1}}$ and the last cluster $k$ is in position $x_{N}$. Therefore, the system is in a jam state.

Suppose the system has reached a jam state. Then, all the clusters are separated by $\nu$ except for the clusters $h$ and $h+1$ which are separated by $\gamma$. As the cars try to move $\nu$, it is easy to see that if the cluster $h+1$ is not empty, all the clusters will remain at the same relative position. If that cluster is empty then only the relative positions of clusters $h, h+1$ and $h+2$ will change from $\pi_{h+1}-\pi_{h}=\gamma, \pi_{h+2}-\pi_{h+1}=\nu$ to $\pi_{h+1}-\pi_{h}=\nu, \pi_{h+2}-\pi_{h+1}=\gamma$, remaining in a jam state.

The function $\delta(x)$ can be seen as a sort of distance to a jam regime and it verifies the following property.

Theorem 1: The sequence $t \mapsto \delta\left(X^{t}\right)$ is non increasing. The proof of the theorem is in Appendix 1.

Theorem 2: A jam regime is almost always reached, i.e., with probability one.

Proof: In order to prove that a jam regime is reachable, we construct a finite sequence of independent events with positive probability after which the system reaches a jam state. Then, this finite sequence will appear with probability one in an infinite sequence of events (Cantelli-Borel).

The dynamics of the system is given by the matrix $C(\omega)=$ $A^{*} B(v(\omega))$, where $B$ is the diagonal matrix of car desiredspeeds chosen randomly and independently between 0 and $\nu$. Let us consider the matrix $C_{j}$ associated with the speed $(0 \cdots 0, \nu, 0 \cdots 0)$ with $\nu$ in position $j$. All the matrices $C_{j}, j=1 \cdots N$ have a strictly positive probability of occurrence.

Consider the finite sequence of independent events associated to the following matrix product, where $k$ is the number of clusters,

$$
C_{1}^{k} C_{2}^{k} \ldots \ldots C_{N-2}^{k} C_{N-1}^{k} .
$$

It is easy to understand why after these events, all the cars are together in only one cluster. The last car $(N)$ stays at the same position, the previous car $(N-1)$ tries to move $k$ times $\nu$ joining the car $N$ and so on. At the end all the cars will be together in only one cluster obtaining a jam state.

The particular jam state used in the proof, has only the property of being easily characterized. Other jam states are reachable with a higher probability.

\section{The Stationary CAR Distribution}

Let us determine the stationary distribution of the population of cars $b=\left(b_{1}, \cdots, b_{k}\right)$ in the $k$ clusters.

Theorem 3: The stationary distribution of $b$ is uniform on the simplex :

$$
B_{N, k}=\left\{b \mid b .1=N, b \in \mathbb{N}^{k}\right\},
$$

where $N$ is the total number of cars, $k$ is the number of clusters in the stationary regime and $\mathbf{1}$ is a k-column vector of 1 .

Proof: Let us consider the Markov chain where the states belong to $B_{N, k}$ having $C_{N}^{N+k-1}$ nodes. Let us show that for each outgoing arc from a node there is an incoming arc with the same transition probability (which shows that the transition matrix is bistochastic).

Outgoing: let us consider the transition from the state $b$ to the state $b^{\prime}$. This state can be written as $b^{\prime}=b-d+\theta d$ where $\theta$ denotes the circular shift of a vector, $\theta: d=\left(d_{1}, \cdots, d_{k}\right) \mapsto$ $\left(d_{k}, d_{1}, \cdots, d_{k-1}\right)$ and $d$ is the leaving cluster vector (this means that there are $d_{j}$ cars that leave the cluster $j$ to the cluster $j+1$ ). The probability of that event is

$$
\lambda^{\sum d_{j}} \mu^{\sum \phi\left(b_{j}-d_{j}\right)} \text { where } \phi(s)= \begin{cases}0 & \text { if } s=0, \\ 1 & \text { otherwise. }\end{cases}
$$




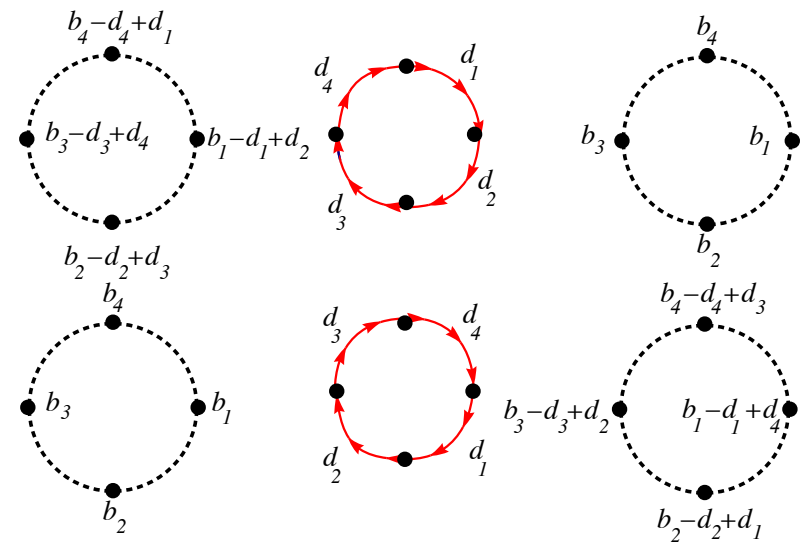

Fig. 4. Equally probable transitions

Incoming: we consider now the state $b-d+\theta^{-1} d$ from which we can reach the state $b$ with $\theta^{-1} d$ as leaving cluster vector. The probability of this event is

$$
\lambda^{\sum d_{j}} \mu^{\sum \phi\left(b_{j}-d_{j}+d_{j+1}-d_{j+1}\right)},
$$

but $\phi\left(b_{j}-d_{j}+d_{j+1}-d_{j+1}\right)=\phi\left(b_{j}-d_{j}\right)$ and thus it has the same probability than the corresponding outgoing arc.

To complete the proof, we have to show that the map that associates to each output arc an input one, is bijective. For that, since the map is injective, let us show that the number of outgoing arcs from a particular state $b$ is equal to the number of incoming arcs to this state. The number of outgoing arcs from $b$ is the number of elements of the set $\{d \mid 0 \leq d \leq b\}$ where the order relation is considered componentwise. The incoming arcs to state $b$ is given by $d^{\prime}$ such that there exists a state $b^{\prime}$ with $b^{\prime}-d^{\prime}+\theta d^{\prime}=b$ and $0 \leq d \leq b^{\prime}$, but this implies that $0 \leq d^{\prime} \leq b$. Therefore, the set of incoming arcs to $b$ is defined by $\left\{d^{\prime} \mid 0 \leq d^{\prime} \leq b\right\}$ which has the same cardinality that the set of outgoing arcs from $b$.

\section{Mean Speed Computation}

The knowledge of the distribution of probability of $n$ allows the explicit computation of the mean speed. We do that in the following theorem.

Theorem 4: For the regular case the mean speed $\bar{v}_{\lambda}(N, k)$ can be obtained recursively as

$$
\bar{v}_{\lambda}(N+1, k)=\frac{\lambda}{N+k}\left(1+N \bar{v}_{\lambda}(N, k)\right),
$$

where $\bar{v}_{\lambda}(1, k)=\lambda \nu$. Moreover for large $N$ we have the asymptotic result

$$
\bar{v}_{\lambda}(N, k)=\frac{\lambda}{N \mu}+o(1 / N) .
$$

Proof: Let us compute the mean speed. Consider a cluster, the first car in the cluster leaves with probability $\lambda$ increasing the mean speed in $\lambda \nu / N$, then the second car leaves this cluster with probability $\lambda^{2}$ increasing the mean speed in $\lambda^{2} \nu / N$ and so on. Then, the mean speed will be $\mathbb{E}(V)$ where

$$
V=\sum_{s=1}^{k}\left(\sum_{j=1}^{b_{s}} \lambda^{j} \frac{\nu}{N}\right)
$$

Developing (16) we obtain

$$
V=\lambda \frac{\nu}{N} \sum_{s=1}^{k} \frac{1-\lambda^{b_{s}}}{1-\lambda}=\frac{\lambda}{\mu} \frac{\nu}{N}\left(k-\sum_{s=1}^{k} \lambda^{b_{s}}\right)
$$

and by linearity

$$
\bar{v}_{\lambda}(N, k)=\mathbb{E}(V)=\frac{\lambda}{\mu} \frac{\nu}{N}\left(k-S_{k}(N)\right),
$$

where we have denoted

$$
S_{k}(N)=\mathbb{E}\left(\sum_{s=1}^{k} \lambda^{b_{s}}\right) .
$$

Using the fact that the probability distribution of $b$ is uniform, we have that

$$
S_{k}(N)=\mathbb{E}\left(\sum_{s=1}^{k} \lambda^{b_{s}}\right)=\sum_{B_{N, k}} \pi_{N, k} \sum_{s=1}^{k} \lambda^{b_{s}},
$$

where we have denoted $\pi_{N, k}=\left(\complement_{N}^{N+k-1}\right)^{-1}$. Interchanging the summation order we obtain

$$
\begin{aligned}
S_{k}(N) & =\pi_{N, k} \sum_{h=0}^{N} \sum_{s=1}^{k} \sum_{B_{N, k}}\left\{\lambda^{h} \mid b_{s}=h\right\} \\
& =\pi_{N, k} \sum_{h=0}^{N} \sum_{s=1}^{k} \complement_{N-h}^{N-h+k-2} \lambda^{h} \\
& =k \pi_{N, k} \sum_{h=0}^{N} \complement_{N-h}^{N-h+k-2} \lambda^{h} .
\end{aligned}
$$

Now, for $N+1$, we have

$$
\begin{aligned}
S_{k}(N+1) & =k \pi_{N+1, k} \sum_{h=0}^{N+1} \complement_{N+1-h}^{N+1-h+k-2} \lambda^{h} \\
& =\frac{k(k-1)}{N+k}+k \pi_{N+1, k} \sum_{h=0}^{N} \complement_{N-h}^{N-h+k-2} \lambda^{h+1} \\
& =\frac{k(k-1)}{N+k}+\frac{N+1}{N+k} \lambda S_{k}(N) .
\end{aligned}
$$

Replacing the recursive formula of $S_{k}$ in (17) we obtain (14). To find the asymptotic result (15) we remark that $S_{k}(N)$ goes to 0 when $N$ goes to $\infty$.

As an example we obtain for $N=3$ and $k=3$ that $\bar{v}_{\lambda}(3,3)=\nu\left(6 \lambda+3 \lambda^{2}+\lambda^{3}\right) / 10$, and for $N=4$ and $k=4$ $\bar{v}_{\lambda}(4,4)=\nu\left(\lambda^{4}+4 \lambda^{3}+10 \lambda^{2}+20 \lambda\right) / 35$.

\section{Extensions and Numerical Results}

The previous analysis of the stochastic model may be done also in the non anticipative case. It can be extended to the case where the cars have a non negligible size $\sigma$. The models are still stochastic min-plus linear, for example in the latter case, we have :

$$
x_{n}^{t+1}= \begin{cases}v_{n}^{t} x_{n}^{t} \oplus(-\sigma) x_{n+1}^{t+1}, & \text { if } n<N, \\ v_{N}^{t} x_{N}^{t} \oplus(1-\sigma) x_{1}^{t+1}, & \text { if } n=N .\end{cases}
$$

Using the formulæ obtained, or a simulator using the MAXPLUS SCILAB toolbox [14] we can plot the fundamental traffic law in the different cases. 


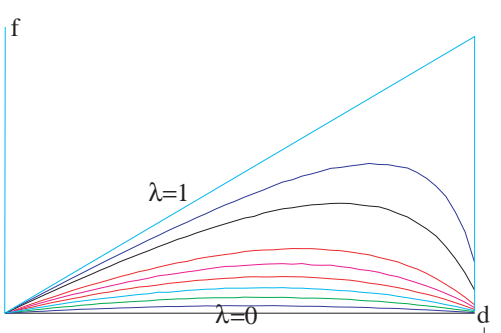

Fig. 5. Flow as a function of the density in the stochastic anticipative case for a continuation of $\lambda$ when $\nu=\sigma$.

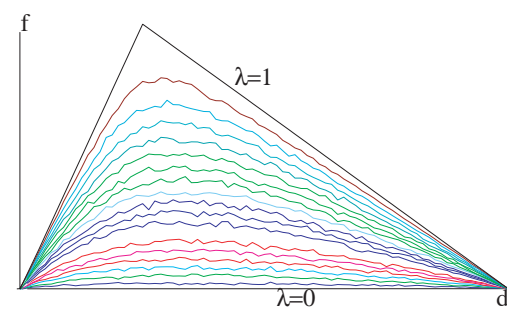

Fig. 6. Flow as a function of the density in the stochastic non anticipative case for a continuation of $\lambda$ when $\nu=3 \sigma$.

\section{CONCLUSION}

For traffic engineers, the main result is the obtainment of a realistic fundamental traffic law shape using a stochastic maxplus linear model defined by four parameters : two possible desired speeds chosen randomly and a security length between the cars. This model can be still more simplified by taking only one desired speed. These models give the typical hat shape of the fundamental traffic law that can be adjusted by choosing these four parameters.

For system engineers, we have given an application of maxplus linear systems. The analysis of the deterministic case is a straightforward application of known results about maxplus algebra. On the other hand, the stochastic case is a rare example where a Lyapunov exponent can be computed explicitly.

\section{APPENDIX: Proof OF THEOREM 1}

Using the following notation for $0 \leq j<l \leq N$,

$$
\Delta_{j}^{l}(x)=\sum_{i=j}^{l-1}\left\{x_{i+1}-x_{i}\right\}
$$

and

$$
\widehat{\Delta}_{j}^{l}(x)=\min _{i}\left(\Delta_{j}^{i}(x)+\Delta_{i+1}^{l}(x)\right),
$$

the function $\delta$ can be written as:

$$
\delta(x)=\widehat{\Delta}_{1}^{N}(x),
$$

where the car numbered $N+1$ is identified with the car 1 . If we call hampered at time $t$ a car $j$ such that $x_{j+1}^{t}-x_{j}^{t}<v_{j}^{t}$, Theorem 1 is an immediate consequence of the following lemma.

Lemma 1: At time $t$, for a sequence $(j+1, \cdots, l-1)$ of hampered cars and for unhampered cars $j$ and $l$ we have :

$$
\Delta_{j}^{l}\left(x^{t+1}\right) \leq \Delta_{j}^{l}\left(x^{t}\right), \quad \widehat{\Delta}_{j}^{l}\left(x^{t+1}\right) \leq \widehat{\Delta}_{j}^{l}\left(x^{t}\right) .
$$

Proof:

i) $\Delta_{j}^{l}\left(x^{t+1}\right) \leq \Delta_{j}^{l}\left(x^{t}\right)$.

Indeed, in this case we have :

$$
\Delta_{j}^{l}\left(x^{t}\right)=\left\{x_{j+1}^{t}-x_{j}^{t}\right\}+x_{l}^{t}-x_{j+1}^{t} .
$$

If car $l$ moves $\nu$, the cars $j+1, \cdots, l-1$ move also $\nu$ and whatever is the desired speed of $j$ we have $\Delta_{i}^{j}\left(x^{t+1}\right)=\Delta_{i}^{j}\left(x^{t}\right)$.

If car $l$ does not move, as $\{a+b\} \leq\{a\}+b$ and car $j$ is unhampered, then :

$$
\Delta_{j}^{l}\left(x^{t+1}\right) \leq\left\{x_{j+1}^{t}-x_{j}^{t}\right\}+x_{j+1}^{t+1}-x_{j+1}^{t}+\Delta_{j+1}^{l}\left(x^{t+1}\right),
$$

moreover, as car 1 does not move we have :

$$
\begin{aligned}
\Delta_{j+1}^{l}\left(x^{t+1}\right) & \leq x_{l}^{t}-x_{j+1}^{t+1} \\
& =x_{l}^{t}-x_{j+1}^{t}-\left(x_{j+1}^{t+1}-x_{j+1}^{t}\right),
\end{aligned}
$$

and the result follows.

ii) Denoting $i$ an index reaching the minimum in the definition of $\widehat{\Delta}_{j}^{l}\left(x^{t}\right)$, we have:

$$
\begin{aligned}
\widehat{\Delta}_{j}^{l}\left(x^{t+1}\right) & \leq \Delta_{j}^{i}\left(x^{t+1}\right)+\Delta_{i+1}^{l}\left(x^{t+1}\right) \\
& \leq \Delta_{j}^{i}\left(x^{t}\right)+\Delta_{i+1}^{l}\left(x^{t}\right)=\widehat{\Delta}_{j}^{l}\left(x^{t}\right) .
\end{aligned}
$$

\section{ACKNOWLEDGMENT}

We are grateful to : $-\mathrm{V}$. Malyshev who propose the stochastic problem, - all the Maxplus group for their useful comments and specially to S. Gaubert who gave us the idea of theorem 2, - the reviewers who help us to improve the redaction.

\section{REFERENCES}

[1] Appert C., Santen L. Modélisation du trafic routier par des automates cellulaires, Actes INRETS 91, Modélisation du trafic, 2002.

[2] F. Baccelli, G. Cohen, G.J. Olsder, J-P. Quadrat, Synchronization and linearity. An algebra for discrete event systems, Wiley and Sons, 1992.

[3] M. Blank, Variational principles in the analysis of traffic flows. (Why it is worth to go against the flow), Markov Processes and Related fields pp.287-305, vol.7, $\mathrm{N}^{\circ} 3,2000$.

[4] Benjaafar S., Dooley K., Setyawan W. Cellular automata for traffic flow modelling. URL: citeseer.nj.nec.com/380458.html

[5] B. Derrida, M.R. Evans, V. Hakim, V. Pasquier, A matrix method of solving an asymmetric exclusion model with open boundaries, pp121133 in Cellular Automata and Cooperative systems Les Houches, France June 1992 eds N. Boccara et al, Kluwer Acad. Publ., 1993.

[6] B. Derrida, M.R. Evans, V. Hakim, V. Pasquier, Exact results for the one dimensional exclusion model Physica A200, 25-33 4th Bar Ilan Conference Frontiers in Condensed Matter Physics, 1993.

[7] B. Derrida, M.R. Evans, Exact steady state properties of the one dimensional asymmetric exclusion model pp. 1-16 in Probability and Phase Transition ed G. Grimmett, Kluwer Ac. Pub., 1994.

[8] Fukui M., Ishibashi Y., Traffic flow in 1D cellular automaton model including cars moving with high speed, Journal of the Physical Society of Japan, vol.65, $\mathrm{N}^{\circ} 6$, pp. 1868-1870, 1996.

[9] S. Gaubert, Performance evaluation of ( $\max$, +) automata, IEEE Trans. on Automatic Control, 40 (12), Dec. 1995.

[10] S. Gaubert, D. Hong, Series expansions of Lyapunov exponents of monotone homogeneous maps, and forgetful monoids, INRIA RR-3971, 2000.

[11] Nagel K., Herrman H.J. Deterministic models for traffic jams, Physica A, 199, pp.254-269, 1993. arXiv:cond-mat/9307064, 2003.

[12] Nagel K., Schreckenberg M., A cellular automaton model for free way traffic, Journal de Physique I, vol.2, $\mathrm{N}^{\circ} 12$, pp. 2221-2229, 1992.

[13] Wang B.-H., Wang L., Hui P.M., Hu B., The asymptotic steady states of deterministic one-dimensional traffic flow models, Physica. B, vol. 279, $\mathrm{N}^{\circ} 1-3$, pp. 237-239, 2000.

[14] http://www-rocq.inria.fr/scilab. 